\title{
Evidence for a Link Between Fkbp5/FKBP5, Early Life Social Relations and Alcohol Drinking in Young Adult Rats and Humans
}

\author{
Ingrid Nylander ${ }^{1} \cdot$ Aniruddha Todkar $^{2} \cdot$ Linnea Granholm $^{1} \cdot$ Maria Vrettou $^{2} \cdot$ \\ Megha Bendre ${ }^{2}$ - Wout Boon ${ }^{2}$ - Henrik Andershed ${ }^{3}$ - Catherine Tuvblad ${ }^{3,4}$. \\ Kent W Nilsson ${ }^{5} \cdot$ Erika Comasco $^{2}$ (D)
}

Received: 21 July 2016 / Accepted: 22 September 2016 /Published online: 5 October 2016

(C) The Author(s) 2016. This article is published with open access at Springerlink.com

\begin{abstract}
Alcohol misuse has been linked to dysregulation of stress, emotion, and reward brain circuitries. A candidate key mediator of this association is the FK506-binding protein (FKBP5), a negative regulator of the glucocorticoid receptor. The aim of the present study was to further understand the Fkbp5/FKBP5-related genetic underpinnings underlying the relationship between early life social relations and alcohol drinking. The effect of maternal separation and voluntary alcohol drinking on Fkbp5 expression was investigated in the brain of young adult rats, whereas the interaction effect of the functional FKBP5 single nucleotide polymorphism rs1360780 genotype and parent-child relationship on problematic drinking was examined in young adult humans. In rats, Fkbp 5 expression in the nucleus accumbens and ventral tegmental area, core regions of the reward system, was affected in a region-dependent manner and in opposite direction by maternal separation and alcohol drinking. Fkbp 5 expression in the cingulate cortex was affected by the combined effect of
\end{abstract}

Electronic supplementary material The online version of this article (doi:10.1007/s12035-016-0157-z) contains supplementary material, which is available to authorized users.

Erika Comasco

Erika.comasco@neuro.uu.se

1 Department of Pharmaceutical Bioscience, Uppsala University, Box 591, SE-75124 Uppsala, Sweden

2 Department of Neuroscience, Uppsala University, Box 593 , SE-75124 Uppsala, Sweden

3 School of Law, Psychology and Social Work, Örebro University, SE-70182 Örebro, Sweden

4 Department of Psychology, University of Southern California, 3620 S. McClintock Ave, Los Angeles, CA 90089-1061, USA

5 Centre for Clinical Research, Västerås Central Hospital, SE-72189 Västerås, Sweden maternal separation and alcohol drinking. In humans, the TT genotype, in the presence of a poor relationship between the child and parents, was associated with problematic drinking behavior. The present findings suggest that $F k b p 5$ expression in mesocorticolimbic dopaminergic regions associates with early life stress-mediated sensitivity to alcohol drinking and that FKBP5 genotype interacts with parent-child relationship to influence alcohol drinking. These findings are the first to point to a role of FKBP5 in propensity to alcohol misuse and call for studies of the underlying molecular mechanisms to identify potential drug targets.

Keywords Alcohol · Brain - Expression · FKBP5 · Genotype $\cdot$ Stress

\section{Introduction}

The FK506-binding protein 5 (FKBP5) has been recently proposed as a drug target for treatment of stress-related disorders $[1,2]$. FKBP5, a co-chaperone of the heat shock protein 90 (Hsp90), can decrease the affinity for glucocorticoids [3, 4] by binding through a tetratricopeptide repeat protein domain on the premature Hsp90 - glucocorticoid receptors (GR) complex and change its conformation $[4,5]$. FKBP5 also promotes the internalization of the immature GR complex, thus reducing the GR complex's activity as a transcription factor [6, 7]. Glucocorticoids, released from the adrenals as a response to a stressor, exert their actions through GRs widespread in the HPA axis, mesocorticolimbic and striatal circuitries. Overlapping expression patterns of Fkbp5 with $\mathrm{Nr} 3 \mathrm{cl}$ (the gene coding for GR) throughout the rodent's brain [8], and its ability as a negative regulator of GR, render FKBP5 a putative target of studies on stress [9] and addiction. 
Early life stress (ELS), in interaction with the genetic make-up, can alter programming of the brain during development [10]. ELS is suggested to interfere with the HPA axis development [11], having been associated with reduced GR expression, negative feedback regulation, and disrupted stress responsivity [12]. ELS, likely through malprogramming of the reward dopaminergic system [13], contributes to vulnerability to alcohol misuse $[12,14,15]$. The interplay between the hypothalamic-pituitary-adrenal (HPA) axis and the mesocorticolimbic and striatal systems is in fact critical for the stress response, emotion processing as well as alcohol seeking and intake $[16,17]$.

Corticosterone, the principal glucocorticoid in rodents, through GRs, increases dopaminergic activity in mesocorticolimbic areas, and reduced GR sensitivity is suggested to increase vulnerability to addiction [18]. Chronic corticosterone administration, which mimics chronic stress, leads to reorganization of the cortical neuronal circuits, likely compromising their top-down inhibitory function exerted on amygdala (Amy) activity [19, 20]. Under stressful conditions, greater Amy activity leads to increased dopamine levels in the nucleus accumbens (Acb) and medial prefrontal cortex (mPFC) possibly through stimulation of the ventral tegmental area (VTA) $[18,19]$.

Alcohol affects the mesocorticolimbic and striatal circuitries as well as the HPA axis response [21, 22]. The initial effect of alcohol is mediated by activation of dopaminergic projections from the VTA to the Acb [17]. The transition from habitual drinking to compulsive drinking involves the dorsal striatum (dStr), mediating the stress-induced seeking and habitual use of alcohol [13], as well as the cortical regions, such as the $\mathrm{mPFC}$ and cingulate cortex $(\mathrm{CCx})$, which are responsible for cognitive control functioning [17]. Projections from the Amy to the HPA axis and Acb mediate the development of negative reinforcement [17]. Nevertheless, the molecular underpinnings of how ELS leads to alcohol misuse are still not well understood [23].

The Fkbp5/FKBP5 genotype in rodents and humans, respectively, has been associated with differences in stress reactivity and alcohol withdrawal [9, 24-26]. A functional polymorphism in the human FKBP5 gene has been identified, SNP rs1360780; homozygosity for the minor allele $\mathrm{T}$ has been associated with twice the amount of FKBP5 protein levels in lymphocytes, relative to the other genotypes [27]. Furthermore, differential stress responsivity, distinctive Amy overreactivity to emotional stimuli as well as psychiatric phenotypes characterized by an impaired stress response have been associated with the T allele or TT genotype, alone or in interaction with aversive environmental factors $[9,28]$.

Poor social interactions during early life, such as poor parent-offspring relationship, constitute a stressor than can influence proneness to alcohol misuse $[10,14]$. To date, the relationship between mesocorticolimbic and striatal Fkbp5 expression and early life stress-mediated propensity to alcohol drinking remains unknown. The present study investigated the effect of maternal separation and adult voluntary alcohol drinking on Fkbp 5 gene expression in the mesocorticolimbic and striatal areas of rats. In the presence of ELS, higher Fkbp 5 gene expression was expected in the Amy, alcohol was hypothesized to counterbalance the effect of ELS on Fkbp 5 gene expression in the $\mathrm{Acb}$, whereas the direction of effects in other brain regions was not predicted. Furthermore, in a translational approach, the interaction effect between FKBP5 rs 1360780 genotype and parent-child relationship on problematic drinking was tested in a population-based sample of young adults. The hypothesis was that drinking problems would be more frequent among carriers of the $\mathrm{T}$ allele whom experienced a poor relationship with their parents.

\section{Methods and Materials}

\section{Study of Rodents}

The study was approved by the Uppsala Animal Ethical Committee (C32/11), according to the Swedish Legislation on Animal Experimentation (Animal Welfare Act SFS1998:56) and the European Communities Council Directive (86/609/EEC). The experiment is described in detail in the supplement and the study outline is presented as Fig. S1. Outbred Wistar male rats (RccHan:WI, Harlan, Europe) were used. Details and previous publications regarding this experiment are reported in the supplementary material.

Early Life Rearing Conditions During the first three postnatal weeks, ELS was simulated by repeated prolonged maternal separations (MS) for $360 \mathrm{~min}$ (MS360), while brief MS with the same handling (15 min; MS15) was applied as control condition.

Voluntary Alcohol Consumption On postnatal week 10, the MS rats were single housed and randomly assigned to water drinking (MS15W, $n=10$; MS360W, $n=10$ ) or free-choice water/alcohol drinking (MS15E, $n=10$; MS360E, $n=20$ ) groups. To simulate human episodic drinking, a voluntary drinking paradigm with repeated drinking and non-drinking days in between was employed [29]. The rats exposed to ethanol had free choice between non-sweetened ethanol (5 or $20 \%$ made from Ethanol $96 \%$; Solveco AB, Rosersberg, Sweden) and water for three consecutive days a week with drug-free days in-between. The first week the rats had free access to $5 \%$ ethanol for $24 \mathrm{~h} /$ day, the next week they had limited access to $5 \%$ for $2 \mathrm{~h} /$ day, and the following five weeks access to $20 \%$ ethanol in $2 \mathrm{~h}$ sessions for three consecutive days a week. Water drinking controls had two bottles with water. After 5 weeks of access to $20 \%$ ethanol and/or water, 
the rats were decapitated immediately after a 2-h drinking session. Trunk blood was collected, and the brain regions VTA, Acb, Amy, dStr, mPFC, and CCx were removed, immediately frozen, and stored at $-80^{\circ} \mathrm{C}$.

Genetic Analyses RNA was isolated and converted to cDNA. Diluted cDNA (20×) was used to assess the expression of Fkbp5 in Amy, Acb, mPFC VTA, dStr, and CCx using CFX96 Touch Real-Time PCR Detection System real-time PCR (BioRad, USA) (Table S1). Relative gene transcripts levels in all animals of each experimental group were determined using the $\triangle \mathrm{CT}$ method (Biorad real time PCR application guide) (Table S2; Fig. S2 and S3).

Corticosterone Measurement Samples were analyzed as described in [30].

\section{Study of Humans}

The Regional Ethical Review Board approved the study (2010/463). Individuals were participants of the Retrospective Study of Young People's Experiences (RESUME), a Swedish population-based, cross-sectional, and retrospective study. Only males were studied since only male rats were investigated, the cohort is described in the Supplement, $(N=838)$. The participants completed a questionnaire and provided a saliva sample and received a small monetary compensation [31]. Details and previous publications regarding this study are reported in the supplementary material.

The Alcohol Use Disorders Identification Test All participants completed the 10-item Alcohol Use Disorders Identification Test (AUDIT) questionnaire regarding quantity (number of standard drinks $=12 \mathrm{~g}$ alcohol), frequency of drinking as well as alcohol-related problems. The results for each question (score range 0-4) were summed to obtain the AUDIT score (i.e., total score range 0-40) (Cronbach's alpha $=0.752$ ). Scoring above eight points in males has been suggested as index of problematic drinking [32].

Parent-Child Relationship Parent-child relationship was measured using the following items: "When you were growing up ( $0-18$ years), how often did your (1) mother, (2) father, show clearly that they liked you, for example, by asking it or giving you a hug or kiss?" These items had a fourpoint response format, ranging from "not very close at all" to "very close." A parent-child relationship summation index (ranging from 0 to 6) was created with a higher score indicating a more positive parent-child relationship. When answering these questions, 98.4 and $95.2 \%$ referred to the biological mother and father, respectively.
Genotyping DNA was extracted from $200 \mu$ of saliva collected with the Oragene self-collection kit (DNA Genotek ${ }^{\circledR}$ ) using the silica-based Kleargene DNA extraction method. Genotyping analyses of the single nucleotide polymorphism (SNP) rs1360780 were performed using the Kbioscience Allele-Specific Polymorphism assay based on competitive allele-specific PCR and bi-allelic scoring. No-template control samples were included to enable the detection of contamination or non-specific amplification.

\section{Statistical Analyses}

Between-group differences were tested using the Mann Whitney $U$ test. The General Linear Model (GLM) test with type III sum of square was used to assess the interaction effect between ELS and drinking, partial eta-squared value were used as effect size index. Group-wise bivariate correlation tests were performed using the Spearman's rank test, and Bonferroni correction for multiple testing was applied. The statistical power of the human study was computed using the Genetic Power Calculator [33]. The size of the populationbased sample had $>80 \%$ of statistical power considering $\alpha=0.05$ and heritability $=1 \%$. The interaction effect of FKBP5 rs1360780 genotype and parent-child relationship on AUDIT was estimated by univariate ANOVA GLM test with type III sum of square test, which allows adjusting for the main effect of the two factors of interest and is considered robust to violations of normality [34]. As confirmatory test, binomial regression was performed, adjusted for the main effect of genotype and environment. For illustrative purposes, fit lines were computed using standard linear regression with the least squares method. All analyses were performed using the Statistical Package for the Social Sciences software v. 21.

\section{Results}

\section{Study of Fkbp5 Expression in Rodents}

In the VTA, water-drinking MS15 rats displayed higher Fkbp 5 expression compared to MS360 rats $(U=14$, $p=.005)$. Alcohol- and water-drinking MS15 rats did not differ in expression levels, whereas alcohol-drinking MS360 rats had higher Fkbp 5 expression than water-drinking MS360 rats $(U=48, p=.045)$ (Fig. 1). In the Acb, alcohol-drinking MS15 rats displayed higher expression of $F k b p 5$ than alcoholdrinking MS360 rats $(U=52, p=.050)$ (Fig. 1). In the CCx, water-drinking MS15 rats $(U=49, p=.024)$, alcohol-drinking MS15 $(U=52, p=.035)$, as well as water-drinking MS360 rats $(U=38, p=.005)$ displayed lower Fkbp5 expression compared to alcohol-drinking MS360 rats (Fig. 1). No group differences were observed on Fkbp 5 expression in the Amy, mPFC, and dStr (data not shown). 

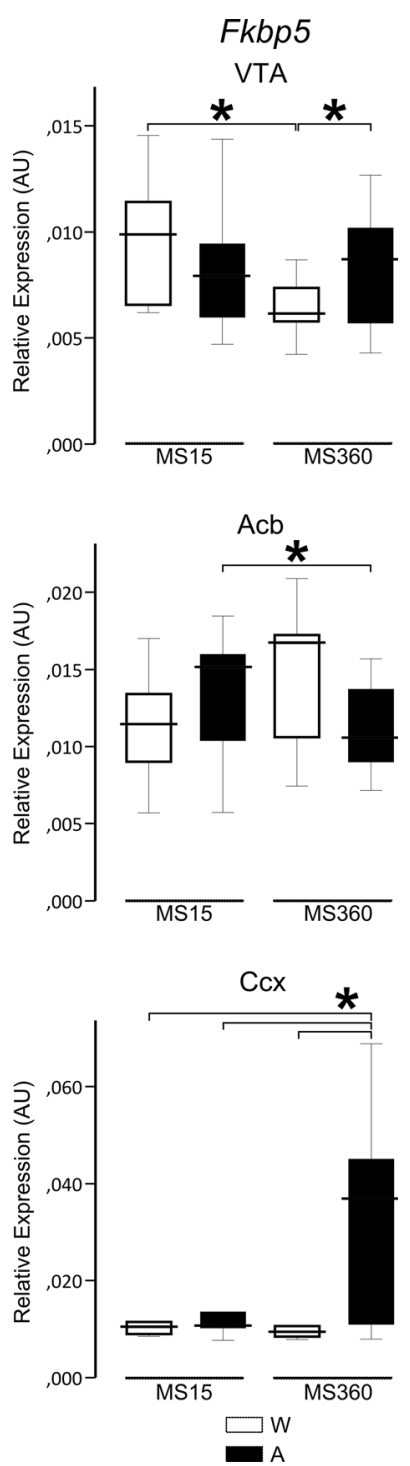

Fig. 1 Median and IQ range of $F k b p 5$ expression in MS rats drinking water or alcohol in a two-bottle free-choice model. $A$ alcohol, $A c b$ nucleus accumbens, $A U$ arbitrary units, $C C x$ cingulate cortex, $M S$ maternal separation (15 or $360 \mathrm{~min}$ ), VTA ventral tegmental area, $W$ water; $* p \leq 0.05$

Interaction effects between ELS and ethanol drinking were observed in the VTA $\left(F=4.35, p=.043, \eta_{p}^{2}=.090\right)$ and Acb $\left(F=5.16, p=.028, \eta_{p}^{2}=.103\right)$ (Fig. S4), but not in other regions except a trend in the $\operatorname{Ccx}(p=0.098)$ (data not shown). Regarding correlations of $F k b p 5$ expression in different regions, a positive correlation was observed between $\mathrm{mPFC}$ and Amy $\left(r_{s}=.740, p<.001\right)$ as well as between mPFC and $\mathrm{CCx}\left(r_{s}=.626, p=.003\right)$ in alcohol-drinking MS360, but not MS15, rats. Correlations that did not pass Bonferroni correction are reported in the Table S3 (non-significant correlations are not reported).

As reported in [30, 35], an interaction effect of ELS and ethanol drinking was found on corticosterone levels with alcohol-drinking MS360 rats displaying lower corticosterone levels compared to alcohol-drinking MS15 as well as waterdrinking MS360 rats. A negative correlation was observed between Fkbp 5 expression in the $\mathrm{mPFC}$ and corticosterone levels in alcohol-drinking MS15 rats $\left(r_{s}=-.943, p=.005\right)$ (Table S3).

Alcohol intake data have been previously reported [30, 36, 37]. The MS15 and MS360 groups did not differ in alcohol consumption although the propensity to increase alcohol intake over time and consume $>1.5 \mathrm{~g} / \mathrm{kg} / 2 \mathrm{~h}$ was more evident in the MS360 group [37], as also reported in several studies [38]. Alcohol intake during the last week before sacrifice as well as the alcohol intake during the last week and last session was not correlated with $F k b p 5$ expression in any brain region or group.

\section{Study of FKBP5 Genotype in Humans}

The mean age of the 838 young male adults was $22.1 \pm 1.4$ (20-24) years for which FKBP5 rs1360780 genotype data were available (HWE $\chi^{2}=0.66, p=0.42$ ), and the age at first intoxication was $15.7 \pm 1.9$ years. The majority reported to drink 2 to 4 times a month, and consumed on average 5-6 drinks containing alcohol in a typical occasion, with AUDIT scores of $7.6 \pm 5.2(0-29)$. They reported that their relationship with their parents until the age of 18 years was $4.3 \pm 1.5$ on a scale between 0 and 6 . Only $3.9 \%$ never lived with both biological parents from birth to the age of 18 , whereas $60.7 \%$ always lived with both biological parents. Though the FKBP5 rs1360780 TT genotype group associated with higher AUDIT scores, FKBP5 rs1360780 genotype frequencies (CC: $53.2 \%$, TC: $40.2 \%$, TT: $6.6 \%$ ) did not differ regarding alcohol intake or relationship with the parents (Table S4). No correlation was observed between AUDIT and parent-child relationship, except among TT carriers for whom problematic drinking was associated with poor parent-child relationship $(r=0.353$; $p<0.008)$. The FKBP5 rs1360780 genotype modulated this association, with carriers homozygous for the $\mathrm{T}$ allele scoring the highest in AUDIT when experiencing a poor relationship with the parents $\left(F_{(2,832)}=3.6 ; p=0.027\right)$ (Fig. 2).

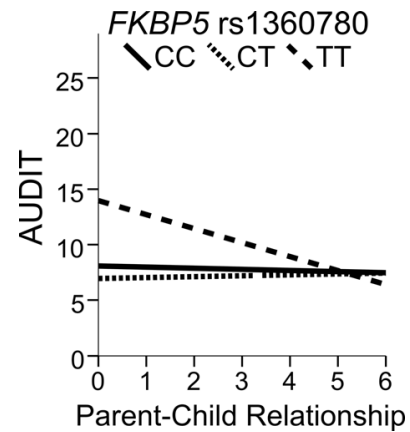

Fig. 2 Fit regression lines between AUDIT score and parent-child relationship ( $0-18$ years) by FKBP5 genotype. A negative slope characterized TT carriers $\left(R^{2}=0.11\right)$ whereas no association was present in $\mathrm{C}$ carriers 


\section{Discussion}

To examine the relation between Fkbp5/FKBP5, early life social interactions and alcohol drinking, a translational approach studying rodents and humans was used. The expression of the Fkbp 5 was assessed in the mesocorticolimbic and striatal areas of young adult outbred rats exposed to an experimental model of ELS and later to an episodic drinking paradigm. The present study for the first time provides mechanistic evidence of long-term effects of maternal separation and also different alcohol-induced effects depending on early life conditions, on Fkbp5 expression in dopaminergic key regions of the reward system in outbred male rats. Moreover, the interaction effect between FKBP 5 rs 1360780 genotype and parentchild relationship on problematic drinking was tested in a population-based sample of young male adults. The TT genotype, in interaction with poor parent-child relationship, was associated with problematic drinking. These findings expand our knowledge on the relations between alcohol drinking, early life social interactions, and $F k b p 5$ expression in rodents as well as FKBP5 genotype in humans.

\section{Fkbp5 Expression in Relation to Maternal Separation and Alcohol Drinking in Rats}

Knowledge on the biological underpinnings mediating the impact of ELS on alcohol misuse is scarce [22, 39]. Disrupted dam-pup interactions during the first postnatal weeks before weaning have been widely advocated as a maladaptive developmental pathway to unfavorable neurobiological and behavioral outcomes [23]. Maternal separation in early life and intermittent voluntary alcohol drinking in young adulthood, two experimental paradigms with high face and construct validity, were herein employed to explore their shaping influence on $F k b p 5$ gene expression. To date, few studies investigated the relationship between Fkbp5 and alcohol drinking and none the interaction with ELS. Intraperitoneal administration of alcohol was shown to increase Fkbp5 expression in the murine striatum putamen and Acb [40], whereas higher withdrawal-like symptoms were displayed by $F k b p 5$ knock-out compared to wild-type mice [26].

ELS associates with long-term molecular changes in the mesocorticolimbic and striatal systems increasing vulnerability to addiction [13]. Herein, the effect of ELS-mediated sensitivity to alcohol drinking on $F k b p 5$ expression localized in the mesocorticolimbic, but not striatal, dopaminergic brain regions. An opposite effect of ELS and alcohol was observed on Fkbp5 expression in the VTA and Acb. In the VTA, rats exposed to ELS through prolonged maternal separation (MS360) had lower Fkbp5 expression than the MS15 rats. Furthermore, alcohol drinking affected $F k b p 5$ expression only in the MS360 rats, which displayed higher levels, towards those seen in the MS15 rats. The gene expression difference in response to voluntary drinking could contribute to the higher propensity for excessive alcohol intake that usually emerges after 3 to 5 weeks of voluntary consumption in MS360 compared to MS15 rats [14]. Interestingly, in the VTA, the direction of the interaction effect was opposite to that in the Acb, likely indicating region-dependent molecular mechanisms involving Fkbp5 in relation to ELS and alcohol in dopaminergic projecting compared to receiving areas, VTA vs. Acb.

In the present study, water-drinking rats exposed to ELS tendentially displayed the highest corticosterone levels [30], which is in line with the long-term impact of maternal separation and the hyporesponsivity of the HPA axis seen in rats exposed to high dam-pup interactions during early postnatal life [23]. Glucocorticoids released as a result of stress have been associated with neuroadaptations in the VTA through direct activation of GR [41], thus supporting a role of FKBP5. Persistent higher corticosterone levels follow prolonged MS in animal models of ELS [42] and are associated with decreased dopaminergic output in the Acb [43]. Since FKBP5 is a negative regulator of GR, the tendentially higher Fkbp 5 expression observed here in the Acb of waterdrinking rats exposed to ELS might be part of a mechanism balancing their higher corticosterone levels. On the other hand, alcohol appeared to reverse these effects. Alcoholdrinking rats exposed to ELS compared to controls displayed lower Fkbp 5 expression in the Acb but higher Fkbp 5 expression in the VTA. Additionally, these rats had lower corticosterone levels [30], in line with the dampening of the stress response by alcohol observed in individuals at risk to develop alcohol use disorder [21].

While the interactive effect between ELS and alcohol seemed to normalize Fkbp 5 expression in the reward circuit to the levels observed in control rats, a unique effect was observed in the CCx. Alcohol-drinking rats exposed to ELS displayed higher Fkbp5 expression compared to all other groups. The $\mathrm{CCx}$ regulates goal-oriented decision-making, outcomes representation and value [44], which are likely to be altered in the presence of ELS and alcohol. Altogether, these findings point to FKBP5 as a modulator of dopaminergic neurotransmission not only in processing of reward and motivational salience but also in executive functioning, supporting the dopamine hypothesis of addiction [45].

As key actors of the dopaminergic system, the VTA and Acb are involved in incentive salience to promote goaldirected behavior, whereas the latter is also part of the extended amygdala complex which integrates the stress-arousal systems with hedonic processing to produce emotional states [17]. Interaction effects could have also been expected in the Amy which is involved in emotion regulation and modulation of the reinforcing effects of alcohol [17]. However, no difference was observed in Fkbp 5 expression in the Amy and not in 
mPFC, another stress-regulating area. Higher Fkbp5 expression has been observed upon restraint stress in the murine Amy as well as in hypothalamic and extra-hypothalamic regions in response to dexamethasone or corticosterone challenge [8, 46]. Knocking out of Fkbp5 in the Amy or the entire brain has been linked to lower fear responses, corticosterone levels, and stress induced fear response, as well as shorter recovery time after chronic social defeat stress in mice [24, 25]. Analysis of the central nucleus of the amygdala, which mediates acute reinforcing actions, separately from the basolateral amygdala, which mediates processes conditioned reinforcing and drug-induced reinstatement [17], might have unveiled differential $F k b p 5$ expression profiles in the present study. A positive fronto-amygdala correlation of Fkbp5 expression was present in alcohol-drinking rats exposed to ELS, suggesting an Fkbp5-mediated interplay between executive and emotional regulating areas under the interactive influence of ELS and alcohol. While the absence of correlations between the VTA, Acb, and Ccx could be interpreted as a consequence of the herein observed effects of maternal separation and alcohol which disrupted potential relationships between these regions. On the other hand, the lack of interaction effects on $F k b p 5$ expression in the mPFC and dStr might be explained by the fact that the rats were sacrificed at a time point when habitual and compulsive stages of alcohol drinking, which are mainly mediated by cortical and striatal regions, were not yet well established. Finally, the strong negative relationship between corticosterone and gene expression in the $\mathrm{mPFC}$ of alcohol-drinking control rats and its absence in other groups calls for further investigation on the underlying mechanism.

\section{FKBP5 Genotype in Relation to Parent-Child Relationship and Problematic Drinking in Humans}

The quality of parent-child interactions can widely vary in humans, from emotional warmth and physical affection to neglect and detachment. This impacts attachment patterns and consequently the emotional and social development of the offspring [47]. Insecure bonding, such as childhood neglect, has been linked to early alcohol initiation and alcohol misuse [47-51]. Early environmental adversity can indeed program the stress response system and imprint brain development with long-term repercussions on neuroplasticity, behavior, and vulnerability to addiction [52]. Nevertheless, not every individual exposed to environmental stressors will misuse alcohol; yet an adverse environment is likely to be a prerequisite for the penetrance of genetic risk factors [15, 52]. Therefore, both constitutional and psychosocial factors are of importance to the risk trajectory that ultimately can culminate into addiction [15].
Signatures of parent-child relationship on both the reward and stress systems are left during development [53]. The association between alcohol misuse and disrupted early life social interactions between the parent and offspring is presumably driven, in part at least, by interactions with genes related to stress reactivity and emotion processing [16]. Parent-child relationship was here studied in relation to FKBP5 genotype and problematic alcohol drinking in young male adults. The TT genotype, in the presence of a poor relationship between the child and the parents, was associated with problematic drinking.

The FKBP5 gene variant examined in the present study has not only genetic [27] but also endocrine and neural functional effects, with the T allele being the vulnerability factor [9]. It is likely that insecurity and negative affect, derived from a poor relationship with the parents, are reasons for alcohol misuse in individuals carrying the more stress-sensitive FKBP5 genotype. Indeed, T/TT carriers have been described to have heightened amygdala reactivity to negative emotional stimuli contingent to emotional neglect $[54,55]$; increased activation during attention bias towards threat and spatial displacement of the hippocampus in the presence of trauma [56]; lower white matter integrity in the posterior cingulum [57] and in the dorsal anterior and posterior cingulate cortex [58], which connect emotional and cognitive processes; weakened connectivity at rest between left amygdala and caudate, parahippocampal gyrus, inferior and middle frontal gyri, in interaction with negative life events [59]; as well as differential gray matter volumes in brain regions involved in cognitive-affective processing $[55,58,60]$, in some cases dependently on experience of adversities [61, 62].

This is to our knowledge the first attempt to investigate the relationship between FKBP5 genotype and alcohol misuse in the context of parent-child relationship. Huang and colleagues demonstrated greater predisposition to develop fewer withdrawal symptoms in patients with alcohol use disorder carrying the T allele [26], a factor that can lead to greater alcohol misuse. More recently, the minor allele of a highly linked SNP, rs9296158, has been associated with reward dependent personality, worse working memory, and blunted autonomic response to stress, among young adults exposed to early life adversity [63]. Binder and colleagues elegantly proved the $T$ allele to enhance FKBP5 gene transcription by enabling the interaction between a glucocorticoid response element and the transcription start site, thus influencing the response to the glucocorticoid receptor activation triggered by early life adversity [64]. All together, these findings provide complementary evidence suggesting a heightened predisposition to stressand emotion-driven alcohol misuse among individuals carrying the T/TT of the FKBP5 rs1360780 SNP.

Addiction is a polygenetic and multifactorial phenotype [65], and several gene-environment association studies have been carried out in the last decades; however, mixed results 
have been found [66]. The present study, by using a relatively large sample, focused on a functional genetic correlate of stress sensitivity and emotion processing $[9,27]$. Yet, the effect of a single genetic polymorphism typically accounts for a minor percentage of the variance in a phenotype [67], and contributes in concert with other factors to explain a certain phenotype, thus the study of stress-related polygenic risk indexes is prompted. Of relevance to the dopaminergic hypothesis of addiction, the interaction between attachment and dopaminergic genotype has been investigated in relation to alcohol use problems in young adults, but no association has been found [68]. Moreover, females should also be studied, since sex differences in stress reactivity and addiction are rather the rule than the exception $[69,70]$; FKBP5 genotype was for instance found to mediate the cortisol stress response in male but not female young adults [71]. The findings on humans have to be understood with respect to these boundaries (see also Strengths and Limitations) and call for independent replications.

\section{Strengths and Limitations}

Experimental animal studies can provide insight into the molecular mechanisms underlying the impact of ELS on brain function and behavior [11, 22]; translational approaches can corroborate them. Similarly, to the human population-based sample, outbred rats were investigated. Episodic voluntary drinking was used to simulate human habitual drinking [29], therefore possessing high face validity. A consumption pattern with repeated drinking days and non-drinking days inbetween is also known to associate with neurobiological changes similar to those seen in the transition from habitual to compulsive drinking [72]. Construct validity, as underlying biology, and predictive validity, as response to alcohol, are also likely to replicate human phenotypic endpoints. Despite differences in the environment (e.g., social stimuli), these characteristics support the translational interpretation of molecular underpinnings of individual variations in vulnerability to alcohol misuse.

Parent-child relationship was employed as proxy of early life interactions between caregiver and offspring; however, the psychometric property of these items is debatable. The attachment construct comprises, among others, bonding typology, parents' responsiveness, and parenting style, which were not considered herein. Moreover, the present data are a representation of the parent-child relationship only from the offspring's perspective. On the other hand, parental deprivation can only be assessed in samples of orphans, though institutional care can be seen as a form of parenting. Almost all individuals spent their first 18 years living with their biological parents, thus strengthening the homogeneity of the present sample and not allowing to investigate the effect of institutional care.
Several unconventional typologies of rearing and caregiving are nowadays common (e.g., single-parent family, adoptive parents, homosexual parents, parents from patchwork families, parents via surrogacy), a factor related to attachment that should be investigated in future studies. Additionally, over the time, the parent-child relationship develops from sensitiveness to visual, tactile and auditory cues, to reciprocity and mutuality, into behavior guidance, parental regard, and independence. The present measure presumably reflects the combination of them, though likely biased by recent memories, since the participants were asked to recall their impression over a period of 18 years.

Notably, causative links could not be estimated in the present cross-sectional study, nonetheless bidirectional influences have also to be expected. Attachment, besides being a predictor of behavior during adulthood and alcohol misuse, has also been shown to be negatively affected by alcohol misuse [51]. Nevertheless, the study of rats points to FKBP5-mediated long-term effects of ELS and its interaction with alcohol consumption. The time point when the rats were sacrificed coincides with the beginning of the establishment of individual alcohol consumption patterns and the beginning of transition into high alcohol intake in the MS360 rats [38]. That is, the rats are not in the addictive state and large differences in alcohol exposure do not confound the results. This serves the purpose to investigate early neurobiological signatures related to the propensity to escalate into excessive drinking. Likewise, the young adults did not yet fulfill the criteria for a diagnosis of AUD (only $2 \%$ scored above 19 on the AUDIT, the threshold for requiring referral to specialist for diagnostic evaluation and treatment); this allowed studying the early stage of the trajectory to addiction. It is indeed of utmost importance to consider early life factors and the period of young adulthood, which is a key developmental period of the brain accompanied by behavioral traits such as risk taking and novelty seeking, and psychosocial factors such as drinking for coping reasons that can predispose to alcohol misuse.

Last but not least, the functionality of the investigated polymorphism, which has been proven not only in terms of protein levels but also HPA axis response, aims to overcome the fact that Fkbp 5 gene expression in vivo cannot be assessed in the human brain. On the other hand, rodents do not carry an orthologous polymorphism. This is therefore an imperfect translational study whose results can be difficult to interconnect and should be seen as a valuable complement to each other.

\section{Conclusion}

The present findings are the first to point to a role of Fkbp5/ FKBP5 in the association between early life social interactions and alcohol consumption in both rodents and humans. The 
mesocorticolimbic, and not striatal, circuit was sensitive to the dual effects of maternal separation and alcohol mediated by Fkbp 5 in rats. FKBP5 genotype modulated the association between parent-child relationship and alcohol misuse in young adults. Future studies should explore Fkbp5-related molecular mechanisms in dopaminergic projecting and receiving areas to advance understanding of the effect of ELS on vulnerability to addiction, causal relationships as well as the effects of drugs targeting FKBP5.

Author Contribution IN: animal experiment design; KWN, HH, CT, AKA: human project design and data collection; EC: present study concept; LG: performing animal experiment; WB, MV, MB: gene expression analyses; EC: genotyping analyses; AT, MV, LG: statistical analyses; EC, AT: drafting the manuscript; IN: critical revision of the manuscript; all authors: finalization and approval of the content of the manuscript.

\section{Compliance with Ethical Standards}

Funding and Disclosure The study was partially supported by funds from the Fredrik and Ingrid Thuring foundation (2012, 2013, 2014), Lars Hierta's Minne foundation (2013), Swedish Brain foundation (PS20130052), Lundberg's and Karlsson's foundation (2013), and Ankarstrand's foundation (2015) to E.C.; from the Alcohol Research Council of the Swedish Alcohol Retailing Monopoly, the European Foundation for Alcohol Research (EA 11 30), the Swedish Research Council (K201261X-22090-01-3) to I.N.; from the Swedish Brain Foundation, the Swedish Alcohol Monopoly Research Council, the Swedish Council for Working Life and Social Research (FAS/FORTE), the Uppsala and Örebro Regional Research Council, the Fredrik and Ingrid Thurings Foundation, the County Council of Västmanland, the KönigSöderströmska Foundation, and the Svenska Spel Research Foundation to K.W.N: and from the National Board of Health and Welfare to H.H (The RESUMÉ data collection). E.C. is a Marie Skłodowska Curie fellow and received funds from the Swedish Research Council (VR: 201500495) and EU FP7-People-Cofund (INCA 600398). The funding body had no role in the design of the study, collection and analysis of data, and decision to publish. The authors declare no conflict of interest.

Open Access This article is distributed under the terms of the Creative Commons Attribution 4.0 International License (http:// creativecommons.org/licenses/by/4.0/), which permits unrestricted use, distribution, and reproduction in any medium, provided you give appropriate credit to the original author(s) and the source, provide a link to the Creative Commons license, and indicate if changes were made.

\section{References}

1. Schmidt MV, Paez-Pereda M, Holsboer F, Hausch F (2012) The prospect of FKBP51 as a drug target. ChemMedChem 7(8):13511359. doi:10.1002/cmdc.201200137

2. Cattaneo A, Riva MA (2015) Stress-induced mechanisms in mental illness: a role for glucocorticoid signalling. J Steroid Biochem Mol Biol. doi:10.1016/j.jsbmb.2015.07.021

3. Binder EB (2009) The role of FKBP5, a co-chaperone of the glucocorticoid receptor in the pathogenesis and therapy of affective and anxiety disorders. Psychoneuroendocrinology 34(Supplement 1):S186-S195. doi:10.1016/j.psyneuen.2009.05.021
4. Wochnik GM, Ruegg J, Abel GA, Schmidt U, Holsboer F, Rein T (2005) FK506-binding proteins 51 and 52 differentially regulate dynein interaction and nuclear translocation of the glucocorticoid receptor in mammalian cells. J Biol Chem 280(6):4609-4616. doi:10.1074/jbc.M407498200

5. Galat A (2013) Functional diversity and pharmacological profiles of the FKBPs and their complexes with small natural ligands. Cell Mol Life Sci 70(18):3243-3275. doi:10.1007/s00018-012-1206-Z

6. Zhang X, Clark AF, Yorio T (2008) FK506-binding protein 51 regulates nuclear transport of the glucocorticoid receptor beta and glucocorticoid responsiveness. Invest Ophthalmol Vis Sci 49(3): 1037-1047. doi:10.1167/iovs.07-1279

7. Grad I, Picard D (2007) The glucocorticoid responses are shaped by molecular chaperones. Mol Cell Endocrinol 275(1-2):2-12. doi:10.1016/j.mce.2007.05.018

8. Scharf SH, Liebl C, Binder EB, Schmidt MV, Muller MB (2011) Expression and regulation of the Fkbp5 gene in the adult mouse brain. PLoS One 6(2):e16883. doi:10.1371/journal.pone.0016883

9. Zannas AS, Binder EB (2014) Gene-environment interactions at the FKBP5 locus: sensitive periods, mechanisms and pleiotropism. Genes Brain Behav 13(1):25-37. doi:10.1111/gbb.12104

10. Maccari S, Krugers HJ, Morley-Fletcher S, Szyf M, Brunton PJ (2014) The consequences of early-life adversity: neurobiological, behavioural and epigenetic adaptations. J Neuroendocrinol 26(10): 707-723. doi:10.1111/jne.12175

11. Pautassi RM, Camarini R, Quadros IM, Miczek KA, Israel Y (2010) Genetic and environmental influences on ethanol consumption: perspectives from preclinical research. Alcohol Clin Exp Res 34(6):976-987. doi:10.1111/j.1530-0277.2010.01172.x

12. Enoch M-A (2011) The role of early life stress as a predictor for alcohol and drug dependence. Psychopharmacology 214(1):17-31. doi:10.1007/s00213-010-1916-6

13. Rodrigues AJ, Leao P, Carvalho M, Almeida OF, Sousa N (2011) Potential programming of dopaminergic circuits by early life stress. Psychopharmacology 214(1):107-120. doi:10.1007/s00213-010-2085-3

14. Nylander I, Roman E (2013) Is the rodent maternal separation model a valid and effective model for studies on the early-life impact on ethanol consumption? Psychopharmacology 229(4):555-569. doi:10.1007/s00213-013-3217-3

15. Enoch MA (2006) Genetic and environmental influences on the development of alcoholism - resilience vs. risk. Ann Ny Acad Sci 1094:193-201. doi:10.1196/annals.1376.019

16. Clarke TK, Treutlein J, Zimmermann US, Kiefer F, Skowronek MH, Rietschel M, Mann K, Schumann G (2008) HPA-axis activity in alcoholism: examples for a gene-environment interaction. Addict Biol 13(1):1-14. doi:10.1111/j.1369-1600.2007.00084.x

17. Koob GF, Volkow ND (2010) Neurocircuitry of addiction. Neuropsychopharmacology 35(1):217-238. doi:10.1038/ npp.2009.110

18. Koob GF, Le Moal M (2001) Drug addiction, dysregulation of reward, and allostasis. Neuropsychopharmacology 24(2):97-129

19. Arnsten AF (2009) Stress signalling pathways that impair prefrontal cortex structure and function. Nat Rev Neurosci 10(6):410-422. doi:10.1038/nrn2648

20. Wellman CL (2001) Dendritic reorganization in pyramidal neurons in medial prefrontal cortex after chronic corticosterone administration. J Neurobiol 49(3):245-253

21. Zimmermann U, Spring K, Kunz-Ebrecht SR, Uhr M, Wittchen HU, Holsboer F (2004) Effect of ethanol on hypothalamicpituitary-adrenal system response to psychosocial stress in sons of alcohol-dependent fathers. Neuropsychopharmacology 29(6): 1156-1165. doi:10.1038/sj.npp.1300395

22. Spanagel R, Noori HR, Heilig M (2014) Stress and alcohol interactions: animal studies and clinical significance. Trends Neurosci 37(4):219-227. doi:10.1016/j.tins.2014.02.006 
23. Curley JP, Champagne FA (2016) Influence of maternal care on the developing brain: mechanisms, temporal dynamics and sensitive periods. Front Neuroendocrinol 40:52-66. doi:10.1016/j.yfrne.2015.11.001

24. Hartmann J, Wagner KV, Liebl C, Scharf SH, Wang X-D, Wolf M, Hausch F, Rein T, Schmidt U, Touma C, CheungFlynn J, Cox MB, Smith DF, Holsboer F, Müller MB, Schmidt MV (2012) The involvement of FK506-binding protein 51 (FKBP5) in the behavioral and neuroendocrine effects of chronic social defeat stress. Neuropharmacology 62(1):332-339. doi:10.1016/j.neuropharm.2011.07.041

25. Attwood BK, Bourgognon J-M, Patel S, Mucha M, Schiavon E, Skrzypiec AE, Young KW, Shiosaka S, Korostynski M, Piechota M, Przewlocki R, Pawlak R (2011) Neuropsin cleaves EphB2 in the amygdala to control anxiety. Nature 473(7347):372-375 http://www.nature.com/nature/journal/v473/n7347/abs/10.1038nature09938-unlocked.html\#supplementary-information

26. Huang M-C, Schwandt ML, Chester JA, Kirchhoff AM, Kao C-F, Liang T, Tapocik JD, Ramchandani VA, George DT, Hodgkinson CA, Goldman D, Heilig M (2014) FKBP5 moderates alcohol withdrawal severity: human genetic association and functional validation in knockout mice. Neuropsychopharmacology 39(8):20292038. doi:10.1038/npp.2014.55

27. Binder EB, Salyakina D, Lichtner P, Wochnik GM, Ising M, Putz B, Papiol S, Seaman S, Lucae S, Kohli MA, Nickel T, Kunzel HE, Fuchs B, Majer M, Pfennig A, Kern N, Brunner J, Modell S, Baghai T, Deiml T, Zill P, Bondy B, Rupprecht R, Messer T, Kohnlein O, Dabitz H, Bruckl T, Muller N, Pfister H, Lieb R, Mueller JC, Lohmussaar E, Strom TM, Bettecken T, Meitinger T, Uhr M, Rein T, Holsboer F, Muller-Myhsok B (2004) Polymorphisms in FKBP5 are associated with increased recurrence of depressive episodes and rapid response to antidepressant treatment. Nat Genet 36(12):1319-1325. doi:10.1038/ng1479

28. Comasco E, Gustafsson PA, Sydsjo G, Agnafors S, Aho N, Svedin CG (2015) Psychiatric symptoms in adolescents: FKBP5 genotypeearly life adversity interaction effects. Eur Child Adolesc Psychiatry 24(12):1473-1483. doi:10.1007/s00787-015-0768-3

29. Palm S, Nylander I (2014) Alcohol-induced changes in opioid peptide levels in adolescent rats are dependent on housing conditions. Alcohol Clin Exp Res 38(12):2978-2987. doi:10.1111/acer.12586

30. Bendre M, Comasco E, Nylander I, Nilsson KW (2015) Effect of voluntary alcohol consumption on Maoa expression in the mesocorticolimbic brain of adult male rats previously exposed to prolonged maternal separation. Transl Psychiatry 5:e690. doi:10.1038/tp.2015.186

31. Cater $\AA$, Andershed A-K, Andershed H (2014) Youth victimization in Sweden: prevalence, characteristics and relation to mental health and behavioral problems in young adulthood. Child Abuse Negl 38: 1290-1302

32. Babor TF, Higgins-Biddle JC, Saunders JB, Monteiro MG (2001) The alcohol use disorders identification test - guidelines for use in primary care

33. Purcell S, Cherny SS, Sham PC (2003) Genetic power calculator: design of linkage and association genetic mapping studies of complex traits. Bioinformatics 19(1):149-150

34. Glass GV, Peckham PD, Sanders JR (1972) Consequences of failure to meet assumptions underlying the fixed effects analyses of variance and covariance. Rev Educ Res 42(3):237-288. doi:10.2307/1169991

35. Todkar A, Granholm L, Aljumah M, Nilsson KW, Comasco E, Nylander I (2015) HPA axis gene expression and DNA methylation profiles in rats exposed to early life stress, adult voluntary ethanol drinking and single housing. Front Mol Neurosci 8:90. doi:10.3389/fnmol.2015.00090

36. Vrettou M, Granholm L, Todkar A, Nilsson KW, WallenMackenzie A, Nylander I, Comasco E (2015) Ethanol affects limbic and striatal presynaptic glutamatergic and DNA methylation gene expression in outbred rats exposed to early-life stress. Addict Biol. doi:10.1111/adb.12331

37. Comasco E, Todkar A, Granholm L, Nilsson KW, Nylander I (2015) Alphalpha 2a-adrenoceptor gene expression and early life stress-mediated propensity to alcohol drinking in outbred rats. Int J Environ Res Public Health 12(7):7154-7171. doi:10.3390/ijerph120707154

38. Nylander I, Roman E (2013) Is the rodent maternal separation model a valid and effective model for studies on the early-life impact on ethanol consumption? Psychopharmacology 229(4):555-569. doi:10.1007/s00213-013-3217-3

39. Pryce CR, Feldon J (2003) Long-term neurobehavioural impact of the postnatal environment in rats: manipulations, effects and mediating mechanisms. Neurosci Biobehav Rev 27(1-2):57-71. doi:10.1016/S0149-7634(03)00009-5

40. Piechota M, Korostynski M, Solecki W, Gieryk A, Slezak M, Bilecki W, Ziolkowska B, Kostrzewa E, Cymerman I, Swiech L, Jaworski J, Przewlocki R (2010) The dissection of transcriptional modules regulated by various drugs of abuse in the mouse striatum. Genome Biol 11(5):R48. doi:10.1186/gb-2010-11-5-r48

41. Daftary SS, Panksepp J, Dong Y, Saal DB (2009) Stress-induced, glucocorticoid-dependent strengthening of glutamatergic synaptic transmission in midbrain dopamine neurons. Neurosci Lett 452(3):273-276. doi:10.1016/j.neulet.2009.01.070

42. Murgatroyd C, Patchev AV, Wu Y, Micale V, Bockmuhl Y, Fischer D, Holsboer F, Wotjak CT, Almeida OF, Spengler D (2009) Dynamic DNA methylation programs persistent adverse effects of early-life stress. Nat Neurosci 12(12):15591566. doi: $10.1038 / \mathrm{nn} .2436$

43. Pacak K, Tjurmina O, Palkovits M, Goldstein DS, Koch CA, Hoff T, Chrousos GP (2002) Chronic hypercortisolemia inhibits dopamine synthesis and turnover in the nucleus accumbens: an in vivo microdialysis study. Neuroendocrinology 76(3):148-157

44. Krawczyk DC (2002) Contributions of the prefrontal cortex to the neural basis of human decision making. Neurosci Biobehav Rev 26(6):631-664

45. Nutt DJ, Lingford-Hughes A, Erritzoe D, Stokes PR (2015) The dopamine theory of addiction: 40 years of highs and lows. Nat Rev Neurosci 16(5):305-312. doi:10.1038/nrn3939

46. Lee RS, Tamashiro KL, Yang X, Purcell RH, Harvey A, Willour VL, Huo Y, Rongione M, Wand GS, Potash JB (2010) Chronic corticosterone exposure increases expression and decreases deoxyribonucleic acid methylation of Fkbp5 in mice. Endocrinology 151(9):4332-4343. doi:10.1210/en.2010-0225

47. Cooper ML, Shaver PR, Collins NL (1998) Attachment styles, emotion regulation, and adjustment in adolescence. J Pers Soc Psychol 74(5):1380-1397

48. Shin SH, Miller DP, Teicher MH (2013) Exposure to childhood neglect and physical abuse and developmental trajectories of heavy episodic drinking from early adolescence into young adulthood. Drug Alcohol Depend 127(1-3):31-38. doi:10.1016/j.drugalcdep.2012.06.005

49. Patock-Peckham JA, Morgan-Lopez AA (2010) Direct and mediational links between parental bonds and neglect, antisocial personality, reasons for drinking, alcohol use, and alcohol problems. Journal of Studies on Alcohol and Drugs 71(1):95-104

50. Anderson AR, Henry CS (1994) Family system characteristics and parental behaviors as predictors of adolescent substance use. Adolescence 29(114):405-420

51. van der Vorst H, Engels RC, Meeus W, Dekovic M (2006) Parental attachment, parental control, and early development of alcohol use: a longitudinal study. Psychology of addictive behaviors : journal of the Society of Psychologists in Addictive Behaviors 20(2):107116. doi:10.1037/0893-164X.20.2.107 
52. Enoch MA (2011) The role of early life stress as a predictor for alcohol and drug dependence. Psychopharmacology 214(1):17-31. doi:10.1007/s00213-010-1916-6

53. Murgatroyd C, Spengler D (2011) Epigenetics of early child development. Frontiers in psychiatry 2. doi:10.3389/fpsyt.2011.00016

54. White MG, Bogdan R, Fisher PM, Munoz KE, Williamson DE, Hariri AR (2012) FKBP5 and emotional neglect interact to predict individual differences in amygdala reactivity. Genes Brain Behav 11(7):869-878. doi:10.1111/j.1601-183X.2012.00837.x

55. Holz NE, Buchmann AF, Boecker R, Blomeyer D, Baumeister S, Wolf I, Rietschel M, Witt SH, Plichta MM, Meyer-Lindenberg A, Banaschewski T, Brandeis D, Laucht M (2015) Role of FKBP5 in emotion processing: results on amygdala activity, connectivity and volume. Brain Struct Funct 220(3):1355-1368. doi:10.1007/s00429-014-0729-5

56. Fani N, Gutman D, Tone EB, Almli L, Mercer KB, Davis J, Glover E, Jovanovic T, Bradley B, Dinov ID, Zamanyan A, Toga AW, Binder EB, Ressler KJ (2013) FKBP5 and attention bias for threat: associations with hippocampal function and shape. JAMA Psychiatry 70(4):392-400. doi:10.1001/2013.jamapsychiatry.210

57. Fani N, King TZ, Reiser E, Binder EB, Jovanovic T, Bradley B, Ressler KJ (2014) FKBP5 genotype and structural integrity of the posterior cingulum. Neuropsychopharmacology 39(5):1206-1213. doi:10.1038/npp.2013.322

58. Fujii T, Ota M, Hori H, Hattori K, Teraishi T, Sasayama D, Higuchi T, Kunugi H (2014) Association between the common functional FKBP5 variant (rs1360780) and brain structure in a non-clinical population. J Psychiatr Res 58:96-101. doi:10.1016/j.jpsychires.2014.07.009

59. Pagliaccio D, Luby JL, Bogdan R, Agrawal A, Gaffrey MS, Belden AC, Botteron KN, Harms MP, Barch DM (2015) Amygdala functional connectivity, HPA axis genetic variation, and life stress in children and relations to anxiety and emotion regulation. $\mathrm{J}$ Abnorm Psychol 124(4):817-833. doi:10.1037/abn0000094

60. Hirakawa H, Akiyoshi J, Muronaga M, Tanaka Y, Ishitobi Y, Inoue A, Oshita H, Aizawa S, Masuda K, Higuma H, Kanehisa M, Ninomiya T, Kawano Y (2016) FKBP5 is associated with amygdala volume in the human brain and mood state: a voxel-based morphometry (VBM) study. Int J Psychiat Clin 20(2):106-115. doi:10.3109/13651501.2016.1144772

61. Pagliaccio D, Luby JL, Bogdan R, Agrawal A, Gaffrey MS, Belden AC, Botteron KN, Harms MP, Barch DM (2014) Stress-system genes and life stress predict cortisol levels and amygdala and hippocampal volumes in children. Neuropsychopharmacology 39(5): 1245-1253. doi:10.1038/npp.2013.327
62. Grabe HJ, Wittfeld K, Van der Auwera S, Janowitz D, Hegenscheid K, Habes M, Homuth G, Barnow S, John U, Nauck M, Volzke H, Schwabedissen HMZ, Freyberger HJ, Hosten N (2016) Effect of the interaction between childhood abuse and rs1360780 of the FKBP5 gene on gray matter volume in a general population sample. Hum Brain Mapp 37(4):1602-1613. doi:10.1002/hbm.23123

63. Lovallo WR, Enoch MA, Acheson A, Cohoon AJ, Sorocco KH, Hodgkinson CA, Vincent AS, Goldman D (2015) Early-life adversity interacts with FKBP5 genotypes: altered working memory and cardiac stress reactivity in the Oklahoma family health patterns project. Neuropsychopharmacology. doi:10.1038/npp.2015.347

64. Klengel T, Mehta D, Anacker C, Rex-Haffner M, Pruessner JC, Pariante CM, Pace TW, Mercer KB, Mayberg HS, Bradley B, Nemeroff CB, Holsboer F, Heim CM, Ressler KJ, Rein T, Binder EB (2013) Allele-specific FKBP5 DNA demethylation mediates gene-childhood trauma interactions. Nat Neurosci 16(1):33-41. doi:10.1038/nn.3275

65. Goldman D, Oroszi G, Ducci F (2005) The genetics of addictions: uncovering the genes. Nat Rev Genet 6(7):521-532. doi: $10.1038 / \operatorname{nrg} 1635$

66. Duncan LE, Keller MC (2011) A critical review of the first 10 years of candidate gene-by-environment interaction research in psychiatry. Am J Psychiatry 168(10):1041-1049. doi:10.1176/appi.ajp.2011.11020191

67. Gratten J, Wray NR, Keller MC, Visscher PM (2014) Large-scale genomics unveils the genetic architecture of psychiatric disorders. Nat Neurosci 17(6):782-790. doi:10.1038/nn.3708

68. Olsson CA, Moyzis RK, Williamson E, Ellis JA, Parkinson-Bates M, Patton GC, Dwyer T, Romaniuk H, Moore EE (2013) Geneenvironment interaction in problematic substance use: interaction between DRD4 and insecure attachments. Addict Biol 18(4):717726. doi:10.1111/j.1369-1600.2011.00413.x

69. Hartwell EE, Ray LA (2013) Sex moderates stress reactivity in heavy drinkers. Addict Behav 38(11):2643-2646. doi:10.1016/j.addbeh.2013.06.016

70. Sharrett-Field L, Butler TR, Reynolds AR, Berry JN, Prendergast MA (2013) Sex differences in neuroadaptation to alcohol and withdrawal neurotoxicity. Pflugers Archiv : European journal of physiology 465(5):643-654. doi:10.1007/s00424-013-1266-4

71. Mahon PB, Zandi PP, Potash JB, Nestadt G, Wand GS (2013) Genetic association of FKBP5 and CRHR1 with cortisol response to acute psychosocial stress in healthy adults. Psychopharmacology 227(2):231-241. doi:10.1007/s00213-012-2956-x

72. Spanagel R (2003) Alcohol addiction research: from animal models to clinics. Best Pract Res Clin Gastroenterol 17(4):507-518 